\title{
Circulating Cytokines and Growth Factors in Acute Cerebral Large Vessel Occlusion-Association with Success of Endovascular Treatment
}

\author{
Gerrit M. Grosse ${ }^{10} \quad$ Christopher Werlein ${ }^{2} \quad$ Nicole Blume $^{1}$ Omar Abu-Fares ${ }^{3} \quad$ Friedrich Götz $^{3}$ \\ Maria M. Gabriel ${ }^{1}$ Johanna Ernst ${ }^{1}$ Andrei Leotescu ${ }^{1}$ Hans Worthmann ${ }^{1}$ Mark P. Kühnel ${ }^{2,4}$ \\ Danny D. Jonigk ${ }^{2,4}$ Christine S. Falk ${ }^{5, *}$ Karin Weissenborn ${ }^{1, *}$ Ramona Schuppner ${ }^{1, *}$
}

\footnotetext{
1 Department of Neurology, Hannover Medical School, Hannover, Germany

2 Institute of Pathology, Hannover Medical School, Hannover, Germany

3 Institute of Diagnostic and Interventional Neuroradiology, Hannover Medical School, Hannover, Germany

${ }^{4}$ Member of the German Center for Lung Research (DZL), Biomedical Research in End-stage and Obstructive Lung Disease Hannover (BREATH), Hannover, Germany

5 Institute of Transplant Immunology, Hannover Medical School, Hannover, Germany
}

Thromb Haemost 2022;122:623-632.

\begin{abstract}
Address for correspondence Gerrit M. Grosse, MD, Department of Neurology, Hannover Medical School, Carl-Neuberg-Str. 1, 30625 Hannover, Germany (e-mail: grosse.gerrit@mh-hannover.de).
\end{abstract}

\section{Abstract}

\section{Keywords}

- cytokines

- growth factors

- ischemic stroke

- large vessel occlusion

- mechanical thrombectomy
Mechanical thrombectomy (MT) is a highly efficient treatment in patients with acute ischemic stroke due to large vessel occlusion (LVO). However, in a relevant proportion of LVO, no sufficient recanalization can be achieved. The composition of cerebral thrombi is highly heterogeneous and may constitute a relevant factor for insufficient reperfusion. We hypothesized that circulating cytokines and growth factors involved in thromboinflammation and platelet activation may be associated with reperfusion status and thrombus composition in patients undergoing MT. An according biomarker panel was measured in plasma specimens taken prior to MT and at a 7-day follow-up. The reperfusion status was categorized into sufficient or insufficient. The composition of retrieved thrombi was histologically analyzed. Differences of baseline biomarker concentrations between insufficient and sufficient reperfusions were highest for interferon (IFN)- $\gamma$, epidermal growth factor, platelet-derived growth factor (PDGF)$\mathrm{AB} / \mathrm{BB}$, and IFN- $\gamma$-induced protein 10 (IP-10/CXCL10). After applying correction for multiple comparisons and logistic regression analysis adjusting for stroke etiology, intravenous thrombolysis, and vascular risk factors, PDGF-AB/BB was identified as an independent predictor of reperfusion status (odds ratio: $0.403 ; 95 \%$ confidence interval: $0.199-0.819)$. Histological analysis revealed that the majority of thrombi had a mixed composition. In conclusion, this study provides the first evidence that cytokines and growth factors are potential effectors in patients undergoing MT for the treatment of acute ischemic stroke.

These authors contributed equally to this work.

received

April 21, 2021 accepted after revision July 1,2021 published online July 5,2021
DOI https://doi.org/ $10.1055 / \mathrm{a}-1544-5431$ ISSN 0340-6245. (c) 2021. The Author(s).

This is an open access article published by Thieme under the terms of the Creative Commons Attribution-NonDerivative-NonCommercial-License, permitting copying and reproduction so long as the original work is given appropriate credit. Contents may not be used for commercial purposes, or adapted, remixed, transformed or built upon. (https://creativecommons.org/ licenses/by-nc-nd/4.0/)

Georg Thieme Verlag KG, Rüdigerstraße 14, 70469 Stuttgart, Germany 


\section{Introduction}

Mechanical thrombectomy (MT) is a highly efficient treatment in patients with acute ischemic stroke due to occlusion of proximal cerebral arteries. ${ }^{1}$ Successful reperfusion is one of the most important predictors of favorable functional outcome after acute stroke. ${ }^{2}$ However, in a relevant proportion of patients with large vessel occlusion (LVO), no sufficient recanalization can be achieved. $^{3}$ Reasons for insufficient recanalization are diverse and include difficult vascular access, vessel wall interactions, and the localization of the culprit occlusion. In the majority of unsuccessful MT, however, the corresponding thrombus can be passed but not (completely) removed. ${ }^{4,5}$ While thrombus length is a known predictor of lack of recanalization after intravenous thrombolysis, ${ }^{6}$ this association is controversial in patients undergoing MT. ${ }^{7,8}$ Rather, there is a growing body of evidence that the composition of thrombi is highly heterogeneous and may constitute a relevant factor for insufficient or futile recanalization. ${ }^{9-11}$ Compared with red blood cell-rich thrombi, those with a high density of platelets are more complex and reveal higher amounts of dense fibrin, von Willebrand factor (VWF), and neutrophil extracellular traps. ${ }^{10}$ Infiltration of leukocytes may constitute a measure of thrombus maturity and thus organization, which in turn leads to higher stability of the thrombus architecture. ${ }^{8}$ These characteristics might explain why platelet-rich thrombi are prone to resist thrombolysis by recombinant tissue-type plasminogen activator (rt-PA) and are more difficult to retrieve via MT. ${ }^{12-14}$

Others and we have previously shown that the ADAMTS13/VWF-axis plays a role in the success of recanalization and clinical outcome in patients undergoing MT and/or intravenous thrombolysis. ${ }^{15-17}$ of note, VWF is released in the course of platelet activation, ${ }^{18}$ which in turn might constitute a factor in thrombus resistance for recanalization therapies. Furthermore, mechanisms of inflammation and thrombosis are closely intertwined. ${ }^{19}$ Diverse circulating cytokines and growth factors are involved in the physiology of platelet activation and thrombus formation. ${ }^{20}$ Likewise, activated platelets secrete inflammatory mediators and thus interact with immune and endothelial cells in terms of a vicious cycle. ${ }^{21}$ Importantly, cytokine release and subsequent immune cell infiltration follow a temporal pattern in thrombus formation and consolidation. ${ }^{11,22}$ Deeper knowledge of clinically relevant mediators in the context of acute stroke due to LVO might thus open avenues for novel diagnostic and therapeutic targets. In this study, we therefore aimed to investigate whether distinct circulating cytokines and growth factors mediating platelet activation and thromboinflammation are associated with thrombus composition and reperfusion status of LVO in patients undergoing MT.

\section{Methods}

\section{Study Population}

A total of 92 patients with acute ischemic stroke due to LVO who underwent MT were prospectively recruited between
March 2018 and August 2019 at Hannover Medical School. Exclusion criteria were refusal of study participation and evidence of a current malignant disease. All patients or proxies provided written informed consent. The ethics committee of Hannover Medical School approved the study (vote no. 7689).

\section{Clinical Data}

Clinical and demographic data including vascular risk factors, concurrent diseases, and medication were collected according to a case report form. Baseline stroke severity was evaluated according to the National Institutes of Health Stroke Scale (NIHSS). Vascular risk factors were subsumed in the Essen Stroke Risk Score (ESRS). Results from imaging including cranial computed tomography (CT) and/or magnetic resonance imaging (MRI) and CT/MR-angiography were considered. Moreover, we collected results of etiological stroke diagnostics including transthoracic and/or esophageal echocardiography, cardiac monitoring, and Doppler/ duplex ultrasound. Process times, i.e., door-to-needle-time and door-to-groin-time were recorded. Reperfusion was graded using the modified Thrombolysis in Cerebral Infarction (mTICI) score ${ }^{23}$ as determined by two independent board-certified neuroradiologists (O.A.-F., F.G.). Evaluation of the mTICI score was done blinded to the clinical and biomarker data. In case of disagreement, consensus was achieved by an individual case discussion. An mTICI score of $2 c$ or 3 in the anterior circulation and MTICI of $2 b$ or 3 in the posterior circulation was defined as sufficient reperfusion and set as primary endpoint. ${ }^{24}$ The modified Rankin Scale $(\mathrm{mRS})^{25}$ was calculated as an estimate of clinical outcome at 90 days (d) after stroke onset according to a telephone interview with the patient or proxy. Favorable clinical outcome was defined as mRS 0-2 or as equal to the premorbid level.

\section{Biomarker Analysis}

Peripheral venous blood was drawn immediately before groin puncture as well as at a follow- up 7 days after baseline. EDTA plasma was stored at $-80^{\circ} \mathrm{C}$ until biomarker measurements. Cytokine and growth factor concentrations were quantified using the Luminex-based MILLIPLEX MAP Human Cytokine/Chemokine Panel (HCYTA-60K-PX38, Merck Millipore, Darmstadt, Germany) in EDTA-Plasma according to the manufacturer's instructions. The following biomarkers have been measured: epidermal growth factor (EGF), interleukin (IL)-1 $\beta$, Eotaxin (CCL11), granulocyte colony-stimulating factor (G-CSF), granulocyte macrophage colony-stimulating factor (GM-CSF), fractalkine $\left(\mathrm{CX}_{3} \mathrm{CL} 1\right)$, interferon (IFN)- $\alpha 2$, IFN- $\gamma$, IL-10, IL-12p40, IL-12p70, IL-13, IL-15, IL-17A, IL-1RA, IL-1 $\alpha$, IL-2, IL-3, IL-4, IL-5, IL-6, IL-7, CXCL8/IL-8, IFN- $\gamma-$ induced protein 10 (IP-10/CXCL10), monocyte chemotactic protein 1 (MCP-1/CCL2), macrophage inflammatory protein $1 \alpha$ (MIP-1 $\alpha$, CCL3), macrophage inflammatory protein $1 \beta$ (MIP-1 $\beta /$ CCL4), tumor necrosis factor (TNF)- $\alpha$, TNF- $\beta$, vascular endothelial growth factor (VEGF)-A, IL-17E/IL-25, IL-17F, IL-18, IL-22, macrophage colony-stimulating factor (M-CSF), monokine induced by IFN- $\gamma$ (MIG)/CXCL9, and platelet- 
derived growth factor (PDGF)-AA, PDGF-AB/BB. Standard curves and concentrations were calculated with Bio-Plex Manager 6.2 software (Bio-Rad Laboratories, Hercules, California, United States).

\section{Histological Analysis}

Cerebral thrombi were retrieved during MT and fixed in 4\% buffered formalin immediately after removal. Specimens were embedded in paraffin. Thereafter, 2 - $\mu \mathrm{m}$ thick sections were cut from the tissue sections followed by histological staining using hematoxylin and eosin (HE) and Elastica van Gieson (EvG) at the Institute of Pathology at Hannover Medical School. Sections were categorized into an erythrocyte-rich (red), platelet-rich (white), or mixed composition and in regard to organization. The histological analysis was performed blinded to clinical data and biomarker results on a routine diagnostic light microscope (BX43, Olympus, Tokyo, Japan). Representative images were acquired with an Olympus CS50 camera (Olympus, Tokyo, Japan) using Olympus cellSens Software (Olympus, Tokyo, Japan) on the above mentioned routine diagnostic light microscope. Image processing was performed using ImageJ software. ${ }^{26}$

\section{Statistical Analysis}

Cytokine concentrations were transformed using the natural logarithm $\left(\log _{e}\right)$ and mean differences with $95 \%$ confidence intervals (CIs) were calculated for group comparisons. Group differences were analyzed using the two-sided Student's $t$-test for normally distributed data or the Mann-Whitney U-test for ordinal data, as appropriate. The Benjamini-Hochberg procedure was applied to correct for multiple testing. The false discovery rate was controlled at $5 \%$. The Chi-square test or Fisher's exact test was used to compare categorical data, as appropriate, and odds ratios (ORs) with $95 \% \mathrm{Cl}$ were calculated. Binary logistic regression analysis was performed with the dichotomized mTICI as the outcome variable and the according biomarker as the exposure variable (unadjusted; model 1) or biomarker, ESRS, ${ }^{27}$ intravenous thrombolysis, and stroke etiology ${ }^{28}$ as the exposure variables (model 2) to obtain OR with $95 \% \mathrm{CI}$. For outcome analysis the dichotomized mRS at 90 days was used as the outcome variable and the according biomarker as the exposure variable (unadjusted; model 1) or biomarker, NIHSS at baseline, ESRS, stroke etiology, and dichotomized mTICI as exposure variables (model 2). The effect of reperfusion status on functional outcome was estimated adjusting for baseline NIHSS, ESRS, stroke etiology, and occlusion site. The according minimal sufficient adjustment sets were identified via the use of causal diagrams. Inter-biomarker correlations were calculated using Pearson's correlation. Statistical analyses were conducted using IBM SPSS Statistics 26 and SAS Enterprise Guide 7.1. Figures were created using GraphPad Prism 9.0.1 and SAS Enterprise Guide 7.1.

\section{Results}

For patients' demographic and clinical characteristics, see - Table 1. Sufficient reperfusion was achieved in 49 of
92 patients. One patient was lost to follow-up at 90 days. In total, 48 patients had a favorable 90-day outcome. Regarding vascular risk factors, no relevant differences were observed between patients with and without sufficient reperfusion. Of note, in the insufficient reperfusion group, more patients received intravenous thrombolysis ( 70 vs. $51 \%$ ). No differences were observed regarding previous treatments. Stroke severity was slightly lower in patients with insufficient compared with sufficient reperfusion (median NIHSS: 14 vs. 16). Patients with sufficient reperfusion achieved a favorable clinical 90-d outcome in $57 \%$ of cases while this was the case in $48 \%$ of patients with insufficient reperfusion $(\mathrm{OR}=1.467 ; 95 \% \mathrm{CI}$ : $0.640-$ 3.359). After adjustment for baseline NIHSS, ESRS, stroke etiology, and occlusion site, sufficient reperfusion was shown to be an independent predictor of a favorable clinical outcome $(\mathrm{OR}=2.889 ; 95 \% \mathrm{Cl}: 1.031-8.094)$.

\section{Biomarker Concentrations and Reperfusion Status}

The following markers had to be excluded from further analyses due to many values below the minimum detection level: GM-CSF, IL-1 $\alpha$, IL-2, IL-3, IL-4, IL-7, IL-12p70, IL-13, IL$17 \mathrm{~A}$, IL-22, MIP-1 $\alpha / \mathrm{CCL} 3$, and TNF- $\beta$. Biomarker values were available for 91 patients at baseline and for 78 patients at the 7-day follow-up. Comparison of circulating biomarker concentrations between the study groups revealed differences of distinct cytokines and growth factors with higher values in patients with insufficient reperfusion. - Fig. 1 depicts mean differences with $95 \% \mathrm{Cl}$ as well as the according $p$-values and critical values after Benjamini-Hochberg correction. Mean differences were highest for IFN- $\gamma$, EGF, PDGF-AB/BB, and CXCL10/IP-10. However, after correction for multiple comparisons, PDGF-AB/BB was the only marker with statistically significant higher values in patients with insufficient reperfusion ( $p=0.002$ ). - Fig. 2 shows boxplots of the distribution of the top analytes within groups and in - Supplementary Table S1 (available in the online version), the untransformed biomarker concentrations are summarized.

In the univariate logistic regression analysis, $\mathrm{PDGF}-\mathrm{AB} / \mathrm{BB}$ yielded an OR of 0.373 per $\log _{e}$ unit increase (95\% CI: $0.190-$ 0.730 ) for sufficient reperfusion. This result was essentially unchanged in the multivariate regression analysis adjusting for stroke etiology, intravenous thrombolysis, and vascular risk factors as subsumed in the ESRS ( $\mathrm{OR}=0.403 ; 95 \% \mathrm{CI}$ : $0.199-0.819$ ). For an overview of the results from the regression analyses regarding all biomarkers, see - Supplementary Table 52 (available in the online version). According to the adjusted regression models, higher concentrations of PDGF-AB/BB, PDGF-AA, EGF, CXCL10/IP-10, and MCP-1/ CCL2 were found to be the strongest predictors of insufficient reperfusion. -Fig. 3 depicts the results of the regression analyses with regard to the top eight analytes, as derived from the group comparison. No relevant group differences were observed at the 7-day follow-up (see - Supplementary Fig. S1, available in the online version). Comparison of biomarker concentrations between patients treated with and without intravenous thrombolysis using rt-PA revealed relevant differences regarding PDGF-AB/BB $(p=0.001)$, PDGF-AA $\quad(p=0.025), \quad$ EGF $\quad(p=0.025), \quad$ CXCL9/MIG 
Table 1 Demographic and clinical characteristics of the study cohort

\begin{tabular}{|c|c|c|c|}
\hline & $\begin{array}{l}\text { Insufficient reperfusion } \\
(n=43)\end{array}$ & $\begin{array}{l}\text { Sufficient reperfusion } \\
(n=49)\end{array}$ & p-Value \\
\hline Age (y) (median [25th-75th percentile]) & $76(64-83)$ & $75(61-82)$ & 0.331 \\
\hline Sex (male; $n(\%))$ & $16(37 \%)$ & $27(55 \%)$ & 0.098 \\
\hline Arterial hypertension, $n(\%)$ & $35(81 \%)$ & $36(73 \%)$ & 0.458 \\
\hline Diabetes mellitus, $n(\%)$ & $16(37 \%)$ & $12(25 \%)$ & 0.256 \\
\hline Dyslipidemia, $n(\%)$ & $13(30 \%)$ & $17(35 \%)$ & 0.664 \\
\hline Adiposity, $n(\%)$ & $11(26 \%)$ & $16(33 \%)$ & 0.499 \\
\hline Coronary heart disease, $n(\%)$ & $8(19 \%)$ & $14(29 \%)$ & 0.330 \\
\hline Previous myocardial infarction, $n$ (\%) & $5(12 \%)$ & $10(20 \%)$ & 0.397 \\
\hline Previous stroke, $n(\%)$ & $8(19 \%)$ & $8(16 \%)$ & 0.790 \\
\hline Nicotine consumption & $14(33 \%)$ & 17 (35\%) & 0.999 \\
\hline ESRS (median [25th-75th percentile]) & $3(2.0-5.0)$ & $3(2.5-5.0)$ & 0.799 \\
\hline Baseline NIHSS (median [25th-75th percentile]) & $14(10-18)$ & $16(12-20)$ & 0.099 \\
\hline Intravenous thrombolysis, $n(\%)$ & $30(70 \%)$ & $25(51 \%)$ & 0.089 \\
\hline Secondary transfer for MT & $23(54 \%)$ & $27(55 \%)$ & 0.877 \\
\hline DNT (if applicable; min) (median [25th-75th percentile]) & $34(23-40)$ & $30(24-37)$ & 0.420 \\
\hline DTG (median; min) (25th-75th percentile) & $68(47-91)$ & $66(31-87)$ & 0.299 \\
\hline Previous platelet inhibition & $14(33 \%)$ & $15(31 \%)$ & 0.999 \\
\hline Previous anticoagulation & $12(28 \%)$ & $18(37 \%)$ & 0.384 \\
\hline Previous statin treatment & $18(42 \%)$ & $21(43 \%)$ & 0.999 \\
\hline Previous antihypertensive treatment & $35(81 \%)$ & 35 (71\%) & 0.330 \\
\hline $\begin{array}{l}\text { Stroke etiology } \\
\text { Large artery atherosclerosis } \\
\text { Cardioembolic stroke } \\
\text { Cryptogenic stroke } \\
\text { Artery dissection }\end{array}$ & $\begin{array}{l}5(12 \%) \\
25(58 \%) \\
12(28 \%) \\
1(2 \%)\end{array}$ & $\begin{array}{l}5(10 \%) \\
29(59 \%) \\
13(27 \%) \\
2(4 \%)\end{array}$ & 0.964 \\
\hline $\begin{array}{l}\text { Occlusion site } \\
\text { Extracranial carotid artery occlusion } \\
\text { Intracranial carotid artery occlusion } \\
\text { Middle cerebral artery occlusion } \\
\text { Basilar artery occlusion }\end{array}$ & $\begin{array}{l}6(14 \%) \\
7(16 \%) \\
30(70 \%) \\
0(0 \%)\end{array}$ & $\begin{array}{l}8(16 \%) \\
6(12 \%) \\
28(57 \%) \\
7(14 \%)\end{array}$ & 0.070 \\
\hline $\begin{array}{l}\text { Favorable } 90 \text {-day outcome (mRS: } 0-2 \text {, or equal to the } \\
\text { premorbid level) }\end{array}$ & $20(48 \%)(n=42)$ & 28 (57\%) & 0.405 \\
\hline
\end{tabular}

Abbreviations: DNT, door-to-needle-time; DTG, door-to-groin-time; ESRS, Essen Stroke Risk Score; mRS, modified Rankin Scale; MT, mechanical thrombectomy; $\mathrm{mTICl}$, modified Thrombolysis in Cerebral Infarction score; NIHSS, National Institutes of Health Stroke Scale.

$(p=0.005)$, G-CSF $(p=0.027)$, IL-1RA $(p=0.029)$, INF- $\alpha 2$ $(p=0.002)$, and Eotaxin $(p=0.011)$. See online -Supplementary Table $\mathbf{S 3}$ (available in the online version) for an according overview on untransformed biomarker concentrations.

\section{Biomarker Concentrations and Clinical Outcome}

Comparing biomarker concentrations between the favorable and unfavorable clinical outcome groups at 90 days revealed relevant differences for baseline IL-6 and IL-10 $(p=0.004$, $p=0.014$ ), with higher values in the unfavorable outcome group which did not pass the critical value after BenjaminiHochberg correction for multiple testing. IL-6 and IL-10 were associated with unfavorable outcome in the univariate regression analysis $\left(\mathrm{OR}_{\mathrm{IL}-6}\right.$ per $\log _{\mathrm{e}}$-unit increase: 1.823 ;
95\% CI: $1.181-2.815$ and $\mathrm{OR}_{\mathrm{IL}-10}$ per $\log _{\mathrm{e}}$-unit increase: 1.439; $95 \% \mathrm{CI}: 1.071-1.935)$. This association was attenuated, however, after adjusting for baseline NIHSS, ESRS, MTICI, and stroke etiology $\left(\mathrm{OR}_{\mathrm{IL}-6}\right.$ per $\log _{\mathrm{e}}$-unit increase: $1.450 ; 95 \% \mathrm{CI}$ : $0.825-2.550$ and $\mathrm{OR}_{\mathrm{IL}-10}$ per $\log _{\mathrm{e}}$-unit increase: $1.102 ; 95 \%$ $\mathrm{CI}$ : 0.761-1.594). Further relevant differences were not observed at baseline. At 7-day follow-up, there was a remaining difference for IL-6 values $(p=0.010)$ regarding 90-day outcome. Untransformed baseline biomarker concentrations in relation to clinical outcome are summarized in - Supplementary Table $\mathbf{S 4}$ (available in the online version).

There were distinct inter-biomarker correlations, particularly involving associations within cytokines/chemokines and growth factors, which are depicted in - Supplementary Fig. $\mathbf{5 2}$ (available in the online version). 


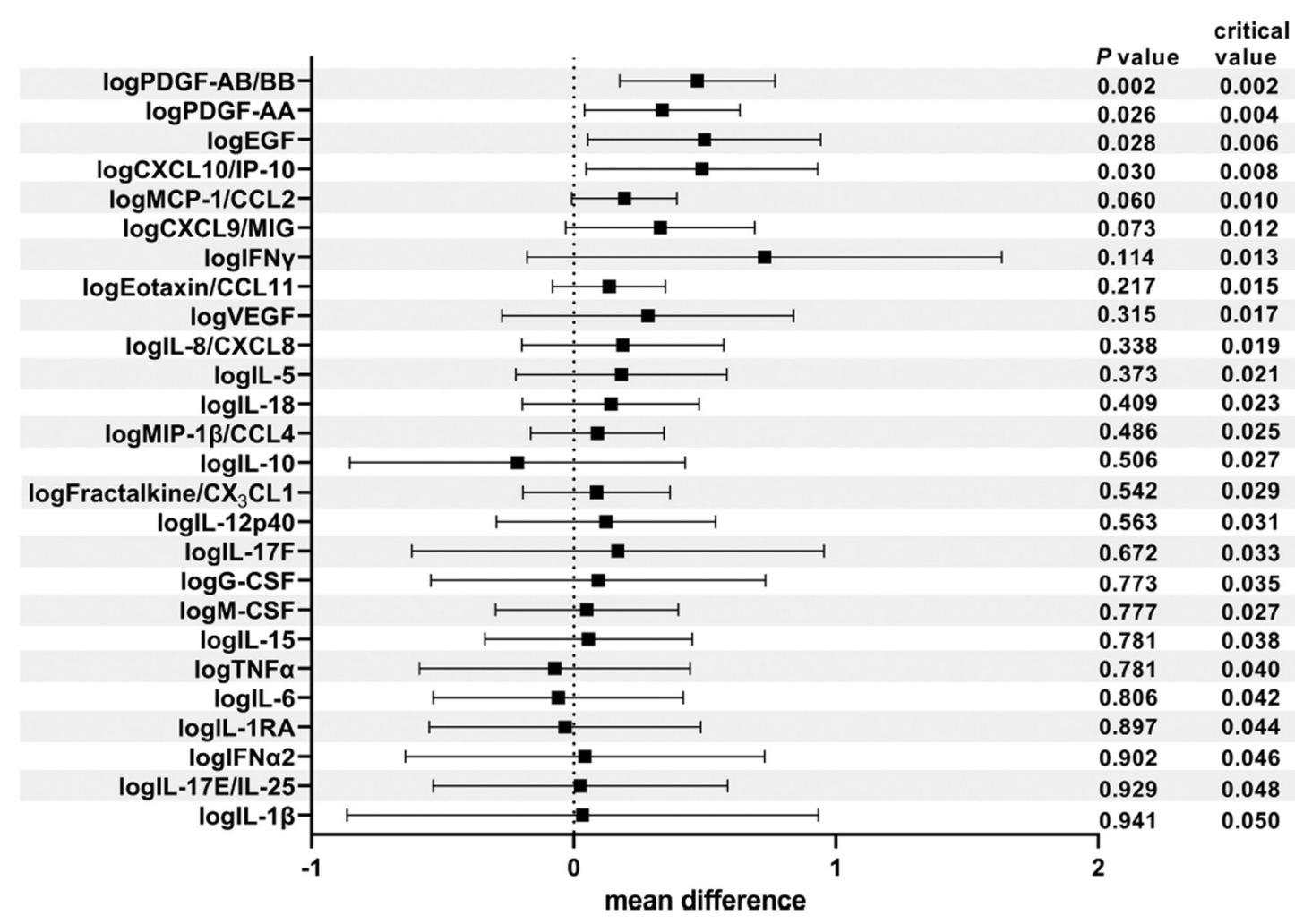

Fig. 1 Mean differences of log-biomarker concentrations between patients with sufficient and insufficient reperfusion. Differences ( $\pm 95 \% \mathrm{Cl}$ ) of mean log-biomarker concentrations between patients with sufficient and insufficient reperfusion. Positive differences refer to higher values in the group to insufficient reperfusion. $p$-Values were calculated with Student's $t$-test. Critical values according to Benjamini-Hochberg correction were calculated via the formula $(i / m)^{*} Q$. $\mathrm{Cl}$, confidence interval.

\section{Histological Analysis}

For 52 cases, a histological analysis of thrombi was possible. In the group of sufficient reperfusion, for $65 \%$ of patients thrombi were available for histological analyses, while this was the case in $46 \%$ with insufficient reperfusion $(\mathrm{OR}=2.17$; $95 \%$ CI: 0.93-5.01). The majority of thrombi revealed a mixed composition, while only nine thrombi could be classified as uniquely erythrocyte-rich (red) and five as platelet-rich (white) (-Supplementary Fig. S3, available in the online version). In the available material, four thrombi showed histological signs of organization (e.g., extracellular matrix deposition; - Supplementary Fig. S4, available in the online version). The distribution of thrombus composition was not different between the study groups. There were no significant differences of biomarker levels regarding the histological group assignments. However, PDGF-AB/BB levels were nonsignificantly higher in patients with mixed or plateletrich thrombi compared with erythrocyte-rich thrombi (-Supplementary Fig. S5, available in the online version).

\section{Discussion}

This study provides first analyses of circulating cytokines, chemokines, and growth factors as potential mediators of recanalization success in patients undergoing MT for treatment of acute LVO. Although MT is a highly effective treat- ment of acute ischemic stroke due to LVO, reperfusion is insufficient in a substantial proportion of patients. Outside clinical trials, TICI scores lower than $2 \mathrm{~b}$ are achieved in $28 \%$ of LVO patients. ${ }^{29}$ Given the impact of reperfusion on functional outcome, ${ }^{24,30}$ however, it was stated that the goal of MT should be to achieve even an mTICI result of at least $2 c$, which therefore was defined as the primary endpoint in our analysis. Consistent with previous reports, patients in whom mTICI $2 c / 3$ could be realized had a higher odds of a favorable functional outcome compared with patients with an mTICI $0-2 b$ in this cohort.

We identified a set of distinct biomarkers with higher baseline concentrations in patients with insufficient reperfusion. Differences between insufficient and sufficient reperfusion status were highest for mean IFN- $\gamma$, EGF, PDGF-AB/BB, and CXCL10/IP-10 levels arguing for a connection to an IFN-mediated inflammation leading to higher secretion of the downstream molecule IP-10/CXCL10 into the circulation. Correction for multiple testing and adjustment for known risk factors of insufficient recanalization were applied. As discussed above, vascular risk factors ${ }^{27}$ and stroke etiology ${ }^{28}$ might confound the efficacy of recanalization therapy and might additionally influence biomarker values. Indeed, in patients treated with intravenous thrombolysis, plasma concentrations of distinct biomarkers were higher than in patients who did not receive intravenous 
A

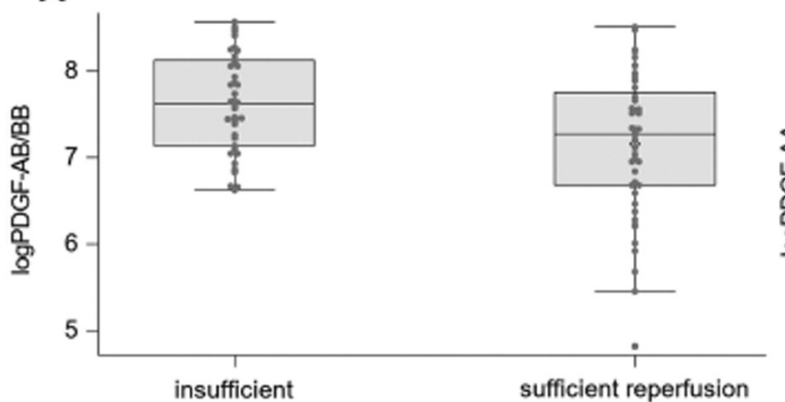

$$
c
$$
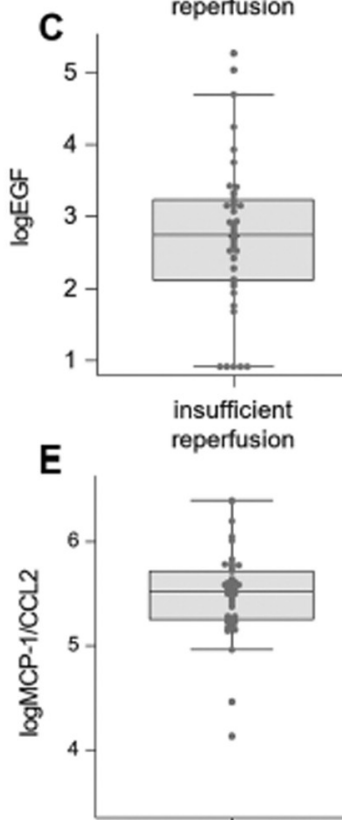

insufficient

G

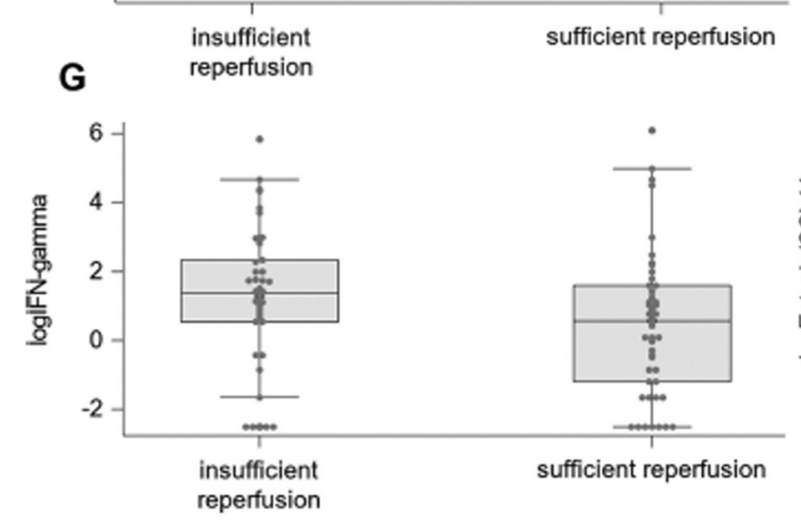

B
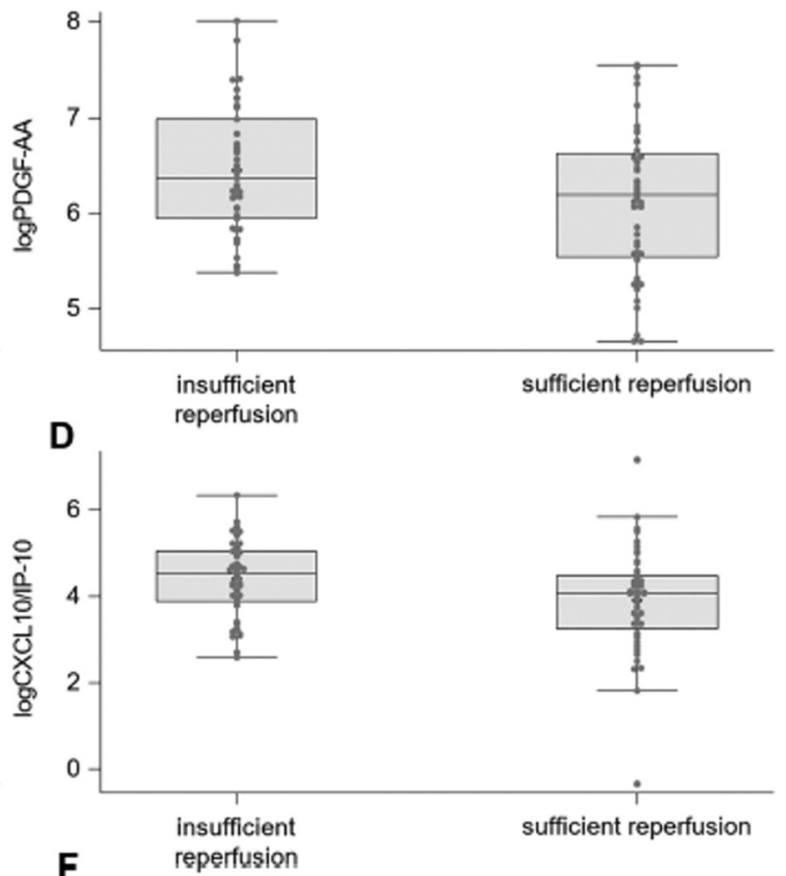

sufficient reperfusion

$F$
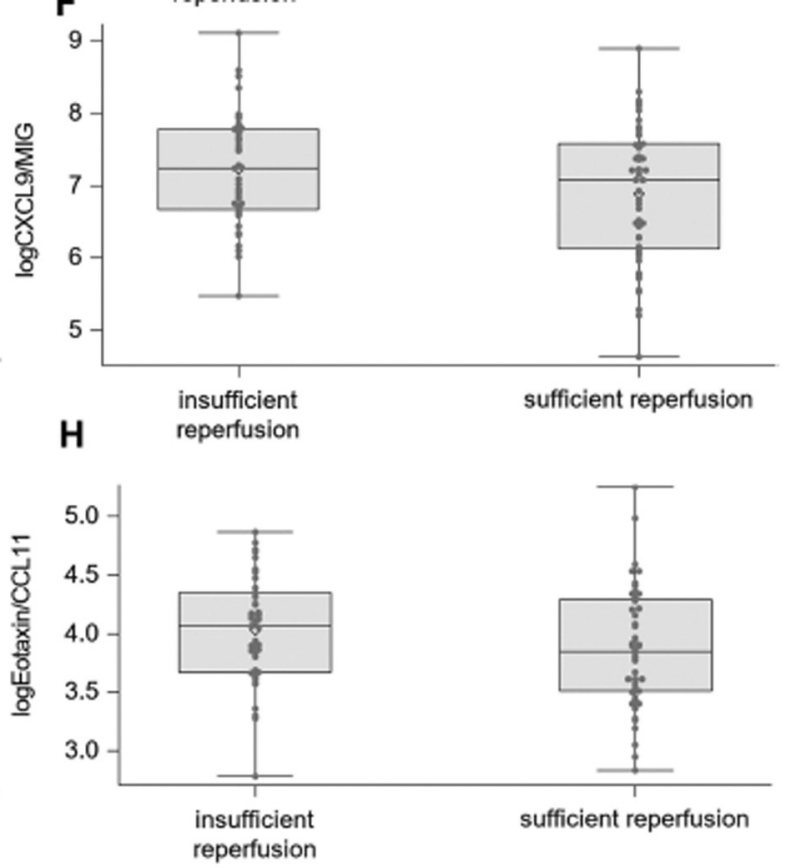

Fig. 2 Distribution of biomarker concentrations in patients with sufficient and insufficient reperfusion. Boxplots depicting log-biomarker values in patients with insufficient $(1 ; \mathrm{TICI} 0-2 \mathrm{~B})$ versus sufficient reperfusion $(2 ; \mathrm{TICI} 2 \mathrm{C}-3)$. In the posterior circulation, $\mathrm{mTICI}$ of $2 \mathrm{~b}-3$ was considered sufficient. (A) PDGF-AB/BB; (B) PDGF-AA; (C) EGF; (D) CXCL10/IP-10; (E) MCP-1/CCL2; (F) CXCL9/MIG; (G) IFN-ץ; (H) Eotaxin/CCL11.

thrombolysis. Thus, we decided to adjust for the ESRS, stroke etiology, as well as intravenous thrombolytic therapy in the regression models. After application of these measures, circulating PDGF-AB/BB was identified as an independent predictor of insufficient recanalization status. PDGF is stored in $\alpha$-granules of platelets and secreted in the course of platelet activation. ${ }^{31}$ PDGF also inherits chemotactic properties on neutrophils and monocytes ${ }^{32}$ and-beside other cytokines and chemokines-is probably facilitating leukocyte recruitment to the culprit thrombus, ${ }^{33}$ making it an interesting target in thromboinflammatory mechanisms. PDGF-BB was moreover implicated in cardiomyocyte injury after recanalization of coronary occlusion. ${ }^{34}$ In a panel of inflammatory cytokines and growth factors, Kim et al recently identified PDGF-AB/BB as the only predictor of progress of intracranial stenosis, ${ }^{35}$ underlining its relevance for atherosclerotic disease. ${ }^{33}$ In this regard, PDGF signaling plays a role in smooth muscle cell proliferation. ${ }^{33}$ 


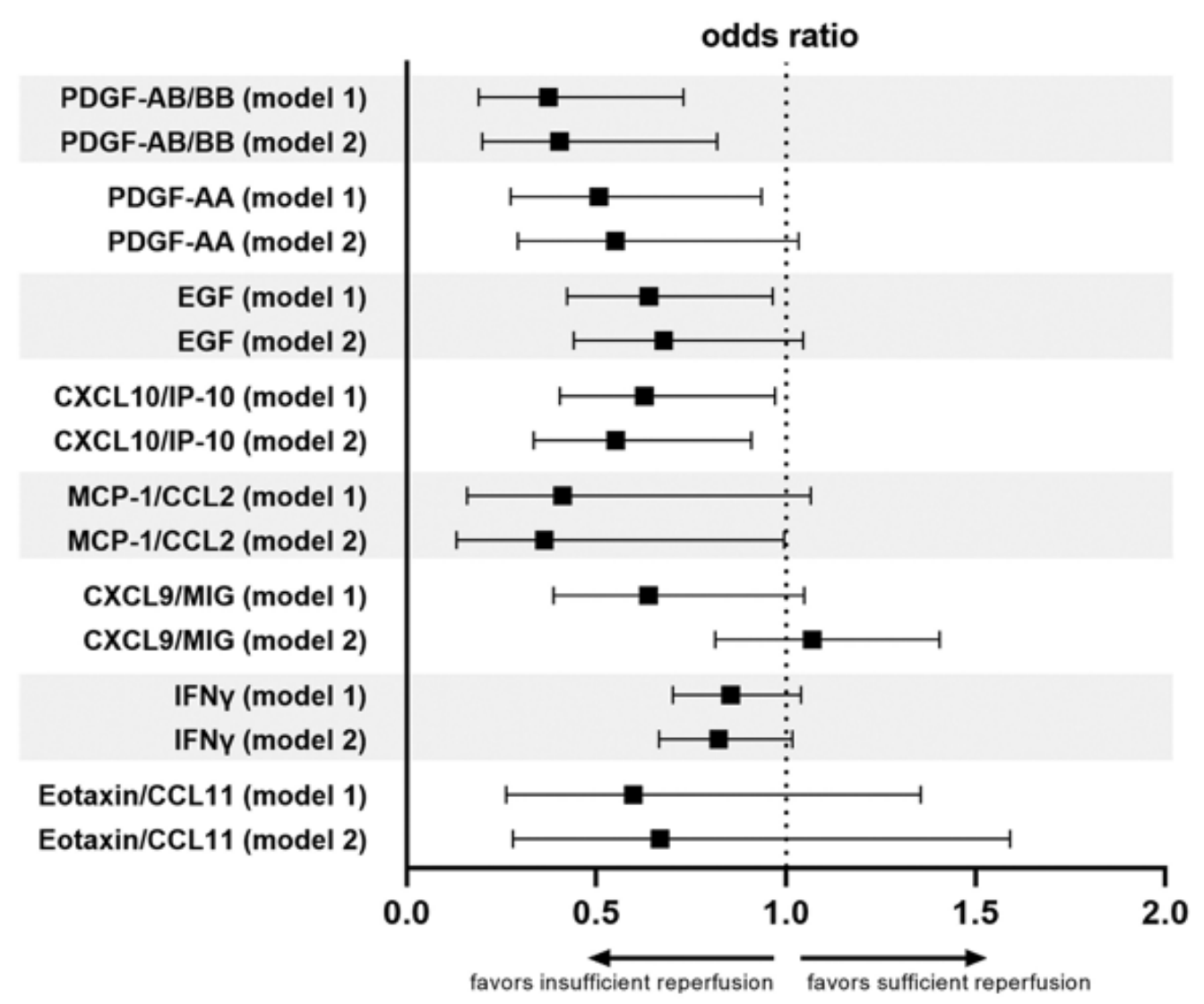

Fig. 3 Results from regression models in predicting reperfusion status. Forest plot indicating unadjusted (model 1 ) and adjusted (model 2 ) odds ratios per $\log _{\mathrm{e}}$-unit increase of biomarker values for sufficient reperfusion.

Notably, there were other mediators associated with insufficient reperfusion in our analysis (e.g., EGF, MCP1/ CCL2, and CXCL10/IP-10), albeit with lower statistical precision. Like PDGF, EGF is stored in $\alpha$-granules of platelets. ${ }^{36}$ MCP-1/CCL2 and CXCL10/IP-10 are established mediators of inflammation and potent chemoattractants of inflammatory cells, primarily monocytes (CCL2) as well as T and natural killer cells (CXCL10), respectively. ${ }^{37,38}$ Interestingly, MCP-1/CCL2 was also implicated in platelet-leukocyte interactions in stroke. ${ }^{39}$ Meanwhile, there is a multitude of data indicating that the interplay of platelets and leukocytes as well as common pathways of inflammation and thrombus formation contributes to brain damage in acute ischemic stroke. ${ }^{40}$ In this context, it is noteworthy that strong evidence exists for circulating MCP-1/CCL2 levels as an independent predictor of the risk for stroke, ${ }^{41}$ which may qualify MCP-1/CCL2 as potential future therapeutic target. ${ }^{38}$ Of interest, various studies could show that PDGF is a potent inductor of MCP-1/CCL2. ${ }^{42}$ In addition, PDGF and MCP-1 lead to induction of tissue factor (TF) and thus promote procoagulant activity on the surface of monocytes. ${ }^{43}$

A prothrombotic role has also been suggested for IFN- $\gamma$, the key cytokine for Th1 T cell responses, which may induce TF synergistically with other mediators of inflammation. ${ }^{44}$ Interestingly, in a murine model of deep vein thrombosis, Nosaka et al demonstrated that IFN- $\gamma$ can negatively affect thrombus resolution. ${ }^{45}$ This hypothesis is supported by further investigations demonstrating that effector memory $\mathrm{T}$ cells produce IFN- $\gamma$ and are crucial in inhibition of thrombus resolution. ${ }^{46-48}$ However, corresponding data in arterial thrombosis and especially in the setting of stroke are lacking in this regard.

Previous histological studies on cerebral thrombi revealed heterogeneous clot characteristics and it has been repeatedly shown that thrombus composition might be a relevant factor for insufficient recanalization..$^{8,10-12,49}$ In accordance with these findings, our analysis also showed a high heterogeneity of thrombus composition with the majority of specimens revealing a mixed architecture. Clear patterns of erythrocyte- or platelet-rich composition were thus rare. Further histologic categorization of mixed specimens might be prone to sampling errors on histologic examination given the high heterogeneity in this cohort and was thus overruled. In addition, there might be a systematic selection bias as the chance of successful thrombus retrieval and thus thrombus analysis was higher in patients with sufficient reperfusion and thrombi with characteristics of resistance to recanalization and/or organization might be under-represented. Histological group comparisons regarding biomarker levels thus suffer from low sample sizes and we were not able to statistically corroborate our hypotheses in this regard. In addition, due to the lower sample size we could not reproduce previous reports that showed an association of thrombus composition with 
reperfusion status. However, PDGF-AB/BB concentrations indeed tended to be higher in patients with platelet-rich thrombi compared with red thrombi, suggesting that further studies with higher sample sizes are merited.

The outcome analysis revealed that baseline IL-6 levels were higher in patients with unfavorable long-term clinical outcome. However, this association was not independent from confounding factors in the multivariable regression analysis. This finding is in line with a previous study from our group investigating another cohort of stroke patients undergoing MT. ${ }^{15}$ A recent investigation by Mechtouff et al stated that IL-6 levels at 24 hours after stroke may be predictive for futile recanalization of LVO. ${ }^{50}$ of note, we did not provide evidence of PDGF being associated with clinical outcome. However, our study might be underpowered for this analysis.

This study has several limitations. Given the multitude of markers investigated, the study cohort is relatively small. We controlled for false-positive findings via Benjamini-Hochberg correction for multiple testing. However, as discussed above, some of the markers shown might be associated with insufficient recanalization, but the respective statistical precision is low in these cases. Importantly, a significant proportion of patients was secondary transferred to our center, leading to application of thrombolytic treatment before blood collection in some of these cases. Thus, intravenous thrombolysis was included in the multivariable models. Furthermore, histological analysis is prone to selection bias and histological groups were too small to derive meaningful conclusions in this regard. Thus, validation of our findings in additional and larger cohorts is clearly needed. In this context, it would also be valuable to investigate to what extent any differences in biomarkers due to stroke etiology influence the recanalization result. Future studies moreover may reveal whether targeting PDGF might constitute a therapeutic strategy in ischemic stroke. Cilostazol is a phosphodiesterase 3 inhibitor that inhibits PDGF release by platelets and is currently used in the treatment of peripheral artery disease. According to recent randomized controlled trials mainly conducted in Asia, cilostazol is also effective and safe in the secondary prevention after stroke. ${ }^{51}$ In a murine model of focal cerebral ischemia, Hase et al could show that cilostazol treatment prevented platelet aggregation, reduced infarct sizes, and improved functional outcome. ${ }^{52}$ Therefore, it would be interesting to investigate whether cilostazol-treated patients might incorporate a higher chance for successful recanalization when suffering an acute stroke due to LVO. It also remains to be shown whether a biomarker-based diagnostic approach may contribute to stratified therapy in an acute setting such as stroke. Hypothetically, circulating cytokines and growth factors could represent interesting candidate markers also in this regard.

\section{Conclusion}

This study presents first investigations of circulating cytokines and growth factors with potential influence on re- canalization success in patients undergoing MT for treatment of acute stroke. Distinct biomarker concentrations were considerably higher in patients with insufficient reperfusion. Of note, these biomarkers possess properties that may be associated with higher resistance to thrombus recanalization. Baseline circulating PDGF-AB/BB was identified as an independent predictor of insufficient reperfusion of LVO. Further studies are warranted to clarify the potential of biomarkers of thromboinflammation and platelet activation for diagnostic and therapeutic approaches in this setting.

\section{What is known about this topic?}

- Although mechanical thrombectomy is a highly efficient treatment of acute ischemic stroke due to large vessel occlusion, reperfusion is insufficient in a substantial proportion of patients.

- Composition of cerebral thrombi is highly heterogeneous and may constitute a relevant factor for insufficient reperfusion.

- Circulating cytokines and growth factors are involved in the physiology of platelet activation and thrombus formation.

\section{What does this paper add?}

- Concentrations of distinct biomarkers measured in plasma samples collected immediately before mechanical thrombectomy are considerably higher in patients with insufficient reperfusion compared with those with sufficient reperfusion.

- Circulating PDGF-AB/BB is an independent predictor of insufficient reperfusion of cerebral large vessel occlusion, after correction for multiple testing and adjustment for confounding factors.

\section{Funding}

This study was supported by PRACTIS-Clinician Scientist Program of Hannover Medical School, funded by the German Research Foundation (grant number: DFG, ME 3696/3-1) (G.M.G.) and by the Clinical Research Group (Klinische Forschergruppe [KFO]) 311 of the German Research Foundation (DFG) (C.W., M.P.K., and D.D.J).

Conflict of Interest

None declared.

\section{Acknowledgments}

The authors like to thank Kerstin Beushausen, Jana Keil, and Christina Petzold for excellent technical assistance.

\section{References}

1 Goyal M, Menon BK, van Zwam WH, et al; HERMES collaborators. Endovascular thrombectomy after large-vessel ischaemic stroke: 
a meta-analysis of individual patient data from five randomised trials. Lancet 2016;387(10029):1723-1731

2 Rha J-H, Saver JL. The impact of recanalization on ischemic stroke outcome: a meta-analysis. Stroke 2007;38(03):967-973

3 Yoo AJ, Andersson T. Thrombectomy in acute ischemic stroke: challenges to procedural success. J Stroke 2017;19(02):121-130

4 Heider DM, Simgen A, Wagenpfeil G, et al. Why we fail: mechanisms and co-factors of unsuccessful thrombectomy in acute ischemic stroke. Neurol Sci 2020;41(06):1547-1555

5 Leischner H, Flottmann F, Hanning U, et al. Reasons for failed endovascular recanalization attempts in stroke patients. J Neurointerv Surg 2019;11(05):439-442

6 Riedel $\mathrm{CH}$, Zimmermann P, Jensen-Kondering U, Stingele R, Deuschl G, Jansen $O$. The importance of size: successful recanalization by intravenous thrombolysis in acute anterior stroke depends on thrombus length. Stroke 2011;42(06):1775-1777

7 Seker F, Pfaff J, Wolf M, et al. Impact of thrombus length on recanalization and clinical outcome following mechanical thrombectomy in acute ischemic stroke. J Neurointerv Surg 2017;9(10): 937-939

8 Yeo LLL, Bhogal P, Gopinathan A, Cunli Y, Tan B, Andersson T. Why does mechanical thrombectomy in large vessel occlusion sometimes fail?: a review of the literature Clin Neuroradiol 2019;29 (03):401-414

9 Heo JH, Nam HS, Kim YD, et al. Pathophysiologic and therapeutic perspectives based on thrombus histology in stroke. J Stroke 2020;22(01):64-75

10 Staessens S, De Meyer SF. Thrombus heterogeneity in ischemic stroke. Platelets 2021;32(03):331-339

11 Jolugbo P, Ariëns RAS. Thrombus composition and efficacy of thrombolysis and thrombectomy in acute ischemic stroke. Stroke 2021;52(03):1131-1142

12 Staessens S, Denorme F, Francois O, et al. Structural analysis of ischemic stroke thrombi: histological indications for therapy resistance. Haematologica 2020;105(02):498-507

13 Maekawa K, Shibata M, Nakajima H, et al. Erythrocyte-rich thrombus is associated with reduced number of maneuvers and procedure time in patients with acute ischemic stroke undergoing mechanical thrombectomy. Cerebrovasc Dis Extra 2018;8 (01):39-49

14 Shin JW, Jeong HS, Kwon H-J, Song KS, Kim J. High red blood cell composition in clots is associated with successful recanalization during intra-arterial thrombectomy. PLoS One 2018;13(05): e0197492

15 Schuppner R, Dirks M, Grosse GM, et al. ADAMTS-13 activity predicts outcome in acute ischaemic stroke patients undergoing endovascular treatment. Thromb Haemost 2018;118(04): 758-767

16 Putzer AS, Worthmann H, Grosse GM, et al. ADAMTS13 activity is associated with early neurological improvement in acute ischemic stroke patients treated with intravenous thrombolysis. J Thromb Thrombolysis 2020;49(01):67-74

17 Bustamante A, Ning M, García-Berrocoso T, et al. Usefulness of ADAMTS13 to predict response to recanalization therapies in acute ischemic stroke. Neurology 2018;90(12):e995-e1004

18 Bryckaert M, Rosa J-P, Denis CV, Lenting PJ. Of von Willebrand factor and platelets. Cell Mol Life Sci 2015;72(02):307-326

19 d'Alessandro E, Becker C, Bergmeier W, et al; Scientific Reviewer Committee. Thrombo-inflammation in cardiovascular disease: an expert consensus document from the Third Maastricht Consensus Conference on Thrombosis. Thromb Haemost 2020;120(04):538-564

20 Koupenova M, Clancy L, Corkrey HA, Freedman JE. Circulating platelets as mediators of immunity, inflammation, and thrombosis. Circ Res 2018;122(02):337-351

21 Mezger M, Nording $H$, Sauter R, et al. Platelets and immune responses during thromboinflammation. Front Immunol 2019; 10:1731-1731
22 Henke PK, Wakefield T. Thrombus resolution and vein wall injury: dependence on chemokines and leukocytes. Thromb Res 2009; 123(Suppl 4):S72-S78

23 Khatri P, Hill MD, Palesch YY, et al; Interventional Management of Stroke III Investigators. Methodology of the Interventional Management of Stroke III Trial. Int J Stroke 2008;3(02):130-137

24 Dargazanli C, Fahed R, Blanc R, et al; ASTER Trial Investigators. Modified thrombolysis in cerebral infarction 2C/thrombolysis in cerebral infarction 3 reperfusion should be the aim of mechanical thrombectomy: insights from the ASTER trial (contact aspiration versus stent retriever for successful revascularization). Stroke 2018;49(05):1189-1196

25 van Swieten JC, Koudstaal PJ, Visser MC, Schouten HJ, van Gijn J. Interobserver agreement for the assessment of handicap in stroke patients. Stroke 1988;19(05):604-607

26 Schindelin J, Arganda-Carreras I, Frise E, et al. Fiji: an open-source platform for biological-image analysis. Nat Methods 2012;9(07): 676-682

27 Goda T, Oyama N, Kitano T, et al. Factors associated with unsuccessful recanalization in mechanical thrombectomy for acute ischemic stroke. Cerebrovasc Dis Extra 2019;9(03):107-113

28 Tiedt S, Herzberg M, Küpper C, et al; GSR Investigators. Stroke etiology modifies the effect of endovascular treatment in acute stroke. Stroke 2020;51(03):1014-1016

29 Deb-Chatterji M, Pinnschmidt H, Flottmann F, et al. Stroke patients treated by thrombectomy in real life differ from cohorts of the clinical trials: a prospective observational study. BMC Neurol 2020;20(01):81

30 LeCouffe NE, Kappelhof M, Treurniet KM, et al; MR CLEAN Registry Investigators $2 \mathrm{~B}, 2 \mathrm{C}$, or 3 : What should be the angiographic target for endovascular treatment in ischemic stroke? Stroke 2020;51(06):1790-1796

31 Kaplan DR, Chao FC, Stiles CD, Antoniades HN, Scher CD. Platelet $\alpha$ granules contain a growth factor for fibroblasts. Blood 1979;53 (06):1043-1052

32 Deuel TF, Senior RM, Huang JS, Griffin GL. Chemotaxis of monocytes and neutrophils to platelet-derived growth factor. J Clin Invest 1982;69(04):1046-1049

33 Rayes J, Bourne JH, Brill A, Watson SP. The dual role of plateletinnate immune cell interactions in thrombo-inflammation. Res Pract Thromb Haemost 2019;4(01):23-35

34 Koizumi T, Kaneda H, Yamasaki T, et al. Impact of platelet-derived growth factor-BB on ischemic myocardial injury after thrombus aspiration for ST-segment elevation myocardial infarction. Int J Cardiol 2015;182:109-111

35 Kim KJ, Jeong SW, Ryu W-S, et al; TOSS-2 Study Group. Plateletderived growth factor is associated with progression of symptomatic intracranial atherosclerotic stenosis. J Clin Neurol 2021; 17(01):70-76

36 Blair P, Flaumenhaft R. Platelet alpha-granules: basic biology and clinical correlates. Blood Rev 2009;23(04):177-189

37 van den Borne P, Quax PHA, Hoefer IE, Pasterkamp G. The multifaceted functions of CXCL10 in cardiovascular disease. BioMed Res Int 2014;2014:893106

38 Bianconi V, Sahebkar A, Atkin SL, Pirro M. The regulation and importance of monocyte chemoattractant protein-1. Curr Opin Hematol 2018;25(01):44-51

39 Franks ZG, Campbell RA, Weyrich AS, Rondina MT. Plateletleukocyte interactions link inflammatory and thromboembolic events in ischemic stroke. Ann N Y Acad Sci 2010;1207:11-17

40 De Meyer SF, Denorme F, Langhauser F, Geuss E, Fluri F, Kleinschnitz C. Thromboinflammation in stroke brain damage. Stroke 2016;47(04):1165-1172

41 Georgakis MK, Malik R, Björkbacka H, et al. Circulating monocyte chemoattractant protein-1 and risk of stroke: meta-analysis of population-based studies involving 17180 individuals. Circ Res 2019;125(08):773-782 
42 Goppelt-Struebe M, Stroebel M. Synergistic induction of monocyte chemoattractant protein-1 (MCP-1) by platelet-derived growth factor and interleukin-1. FEBS Lett 1995;374(03):375-378

43 Ernofsson M, Siegbahn A. Platelet-derived growth factor-BB and monocyte chemotactic protein-1 induce human peripheral blood monocytes to express tissue factor. Thromb Res 1996;83(04): 307-320

44 Nakagomi A, Freedman SB, Geczy CL. Interferon-gamma and lipopolysaccharide potentiate monocyte tissue factor induction by C-reactive protein: relationship with age, sex, and hormone replacement treatment. Circulation 2000;101(15):1785-1791

45 Nosaka M, Ishida Y, Kimura A, et al. Absence of IFN- $\gamma$ accelerates thrombus resolution through enhanced MMP-9 and VEGF expression in mice. J Clin Invest 2011;121(07):2911-2920

46 Najem MY, Couturaud F, Lemarié CA. Cytokine and chemokine regulation of venous thromboembolism. J Thromb Haemost 2020;18(05):1009-1019
47 Luther N, Shahneh F, Brähler M, et al. Innate effector-memory Tcell activation regulates post-thrombotic vein wall inflammation and thrombus resolution. Circ Res 2016;119(12):1286-1295

48 Mukhopadhyay S, Johnson TA, Duru N, et al. Fibrinolysis and inflammation in venous thrombus resolution. Front Immunol 2019;10:1348-1348

49 Goebel J, Gaida BJ, Wanke I, et al. Is histologic thrombus composition in acute stroke linked to stroke etiology or to interventional parameters? AJNR Am J Neuroradiol 2020;41(04):650-657

50 Mechtouff L, Bochaton T, Paccalet A, et al. Association of interleukin-6 levels and futile reperfusion after mechanical thrombectomy. Neurology 2021;96(05):e752-e757

51 Tan $\mathrm{CH}$, Wu AG, Sia CH, et al. Cilostazol for secondary stroke prevention: systematic review and meta-analysis. Stroke Vasc Neurol 2021;6(03):410-423

52 Hase Y, Okamoto Y, Fujita Y, et al. Cilostazol, a phosphodiesterase inhibitor, prevents no-reflow and hemorrhage in mice with focal cerebral ischemia. Exp Neurol 2012;233(01):523-533 